\title{
Combined Mie Resonance Metasurface for Wideband Terahertz Absorber
}

\author{
Jie $\mathrm{Hu}{ }^{1,2}$, Tingting Lang ${ }^{1, *}$, Changyu Shen ${ }^{1}$ and Liyang Shao ${ }^{2}$ \\ 1 Institute of Optoelectronic Technology, China Jiliang University, Hangzhou 310018, China; \\ s1604080303@stu.cjlu.edu.cn (J.H.); shenchangyu@cjlu.edu.cn (C.S.) \\ 2 Department of Electrical and Electronic Engineering, Southern University of Science and Technology, \\ Shenzhen 518055, China; shaoly@sustc.edu.cn \\ * Correspondence: langtingting@cjlu.edu.cn
}

Received: 15 August 2018; Accepted: 15 September 2018; Published: 17 September 2018

\begin{abstract}
In this paper, we propose a combined metasurface consisting of an aluminum substrate and an array of $\mathrm{TiO}_{2}$ blocks to achieve a wideband terahertz absorber. We incorporated several similar dielectric blocks with different side length into each unit cell. Each dielectric block could cause magnetic-resonance-inducing absorption effect with different peak wavelengths. Thus, our combined metasurface could achieve wider absorption frequency band than the traditional design when these dielectric blocks were properly designed. The absorption bandwidth could be widened nearly 2.5 times and 5 times compared to a single block case when there were four and nine blocks, respectively, andcouldbe further improved by increasing the number of combinations in structures (variable parameters included number, spacing, dimensions etc.). For both $\mathrm{TE}_{00}$ (the electric fields of the light polarized along the $y$-axis) and $\mathrm{TM}_{00}$ (the electric fields of the light polarized along the $x$-axis) polarization states, the absorption bandwidth could be widened effectively; even when the incident angle was $45^{\circ}$, the absorption rate could still reach about $75 \%$. This structure is simple and easy to fabricate, and this design concept can also be used in various other application fields.
\end{abstract}

Keywords: absorption; metamaterials; resonance

\section{Introduction}

The use of absorbers can reduce or eliminate the interference of complex electromagnetic environment on wireless communications. Furthermore, the research on absorbers also plays an important role in solar cells [1,2], photodetectors [3], military stealth, and radar detection [4,5]. There have been a number of designs for absorbers, such as multilayer gratings [6] and double-cavity photonic crystal [7], among others.

Many applications based on metasurfaces [8-11] have been realized, such as polarization conversion [12], antireflection coating [13], ultralens [14], etc. Absorbers based on metasurface are also an interesting application and has attracted the attention of many scholars [15-17]. The working range of the metamaterial absorbers can be designed in the visible [15,16], near-infrared [18,19], mid-infrared [20], terahertz [21], and microwave bands [19,22]. A good absorber not only requires high absorption but also a relatively wide operating bandwidth. The insensitivities of absorbers to the polarization and incident angle of incident light are desired characteristics as well. Traditional metamaterial absorbers are based on the electromagnetic dipole of the resonator, which limits the working frequency band of the absorber. Current metamaterial absorbers mostly use a multilayer structure to achieve a wide absorption bandwidth or multiband tunable absorption [23]. Metasurface absorbers based on Salisbury Screen [22] have also been reported. However, these thick and bulky multilayered structures make it difficult for the absorbers to integrate with commercial 
technologies, and the manufacturing process is usually expensive. Synthetic fractals inherently carry spatially encoded frequency information, thus becoming ideal candidates for broadband optical structures. Many metasurface absorbers based on fractals have been proposed recently [24,25]. Mitchell Kenney et al. [24] proposed a structure where self-similar shapes were scaled down in size and added onto the original shape, thus increasing the effective perimeter of the element and achieving a resonance frequency shift. Similarly, M. R. I. Faruque et al. [26] proposed a leaf vein shaped structure. In this case, only the filling factor had changed and the overall size changed very little. Therefore, fractal provides a new degree of freedom-independent of the traditional resonator scale rule-to realize the change of resonant frequency. However, the increase in fractal order will inevitably lead to complexity and the need for precision of the cell structure, which requires advanced manufacturing process. The major disadvantages of fractals are the complicated design and fabrication processes.

As far as we know, although the idea of combining different structures into one unit cell has appeared in other fields, we rarely see reports that widen the absorption bandwidth based on this scheme. In this paper, we propose a wideband terahertz metasurface absorber based on a simple structure that combines resonators of the same shape and different sizes in one unit cell to broaden the absorption bandwidth of the metamaterial absorber. Our metasurface effectively broadened the absorption bandwidth of conventional Mie resonance absorbers. The absorption bandwidth could still be widened when the polarization of the incident light changed; when the incident angle was $45^{\circ}$, the absorption rate could still reach about $75 \%$.

\section{Structure Design and Simulation}

The schematic diagram of our combined Mie resonance metasurface is shown in Figure 1a, and the enlarged view of one unit cell (circled by a yellow box) is shown in Figure 1b. The dielectric blocks were arranged periodically on the aluminum reflective layer. Here, nine dielectric blocks were incorporated into one unit cell. The virtual yellow dashed line in Figure $1 \mathrm{~b}$ (this is for illustration only and was not in the structure) divides the unit cell into nine spaces-similar to the Sudoku. The nine blocks were located at the center of each of these spaces. The side lengths of the square blocks in the $x-y$ plane $\left(\mathrm{a}_{1} \sim \mathrm{a}_{9}\right)$ were from $31 \mu \mathrm{m}$ to $35 \mu \mathrm{m}$, with a step of $0.5 \mu \mathrm{m}$. The periods in both $x$ and $y$ directions were maintained to be $p_{x}=p_{y}=120 \mu \mathrm{m}$. The thickness of the blocks were all $t=30 \mu \mathrm{m}$, and the thickness of the metallic layer for reflection was $0.5 \mu \mathrm{m}$. As metal is a perfect electrical conductor in the terahertz band as their properties are similar, we could have used any kind of metal. At the same time, the material of the structure is not limited to titanium dioxide, and other dielectric with high refractive index, such as silicon or barium strontium titanate (BST), are also feasible. We chose $\mathrm{TiO}_{2}$ in this paper to get larger frequency interval between the fundamental mode and the higher-order mode so that it would be convenient for our analysis. In manufacturing, the substrate under the metal reflective layer can be a quartz substrate or a silicon wafer; this only plays a role as support because there will be no transmission through the metal layer. The dielectric blocks that act as resonators were constructed from titanium oxide $\left(\mathrm{TiO}_{2}\right)$ with a relatively large permittivity of 114 and a loss tangent of 0.01 at terahertz frequencies [27]. The spectra of the absorbers were numerically calculated using the commercial simulation software CST Microwave Studio (CST Studio Suite 2014, CST Company, Darmstadt, Germany) with the finite-element frequency-domain solver. The calculation procedure was similar to what other people in our research group had previously done in their papers [28,29]. Periodic boundary conditions were used in both $x$ - and $y$-directions, while open boundary condition was used in $\pm z$ direction. Both normal-incident $\mathrm{TE}_{00}$ (the electric fields of the light polarized along the $y$-axis) and $\mathrm{TM}_{00}$ (the electric fields of the light polarized along the $x$-axis) modes were defined at the receiving ports to ensure that any polarization conversion was accounted for. At least four mesh steps per wavelength were used to ensure the accuracy of the calculated results. The absorption was obtained by subtracting the transmission and reflection from 1 . As for the transmission and reflection, they could be directly obtained in the S-parameters, which were calculated by the software. 
We assumed the plane wave, with a wave vector of $k$, propagated along the opposite direction of the $z$-axis. The magnetic and electric fields of the light were polarized along the $x$ and $y$ axes, respectively.

(a)

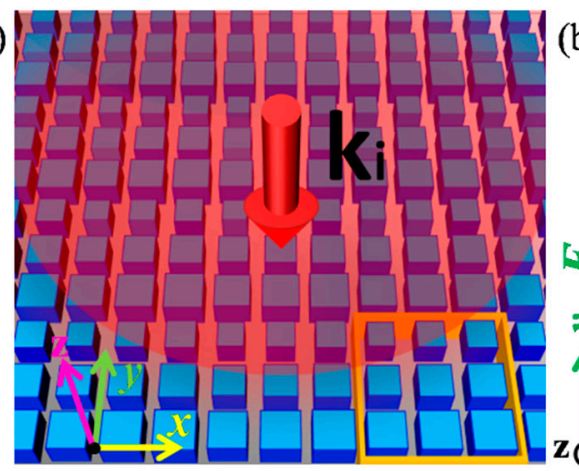

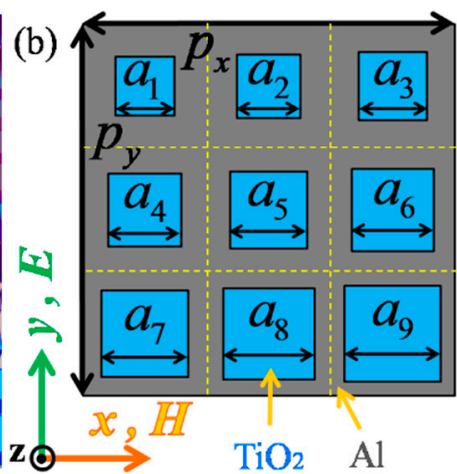

Figure 1. (a) Schematic diagram of our combined metasurface absorber and (b) an enlarged view of one unit cell (circled by a yellow box in Figure 1a).

Figure 2 shows the absorption (black curve), transmission (red curve), and reflection (blue curve) spectra of our combined Mie resonance metasurface. The transmission could be negligible to zero because of the metal layer. The reflection and absorption added up to 1 . The absorption was stable at a high level with a few fluctuations within a certain frequency range, while the spectra of traditional dielectric resonators usually had the Gaussian shape, as shown in Figure 3. Three absorption peaks and two absorption valleys were labeled as "P1", "P2", "P3", "V1", and "V2", respectively, and are pointed with green arrows in Figure 2.

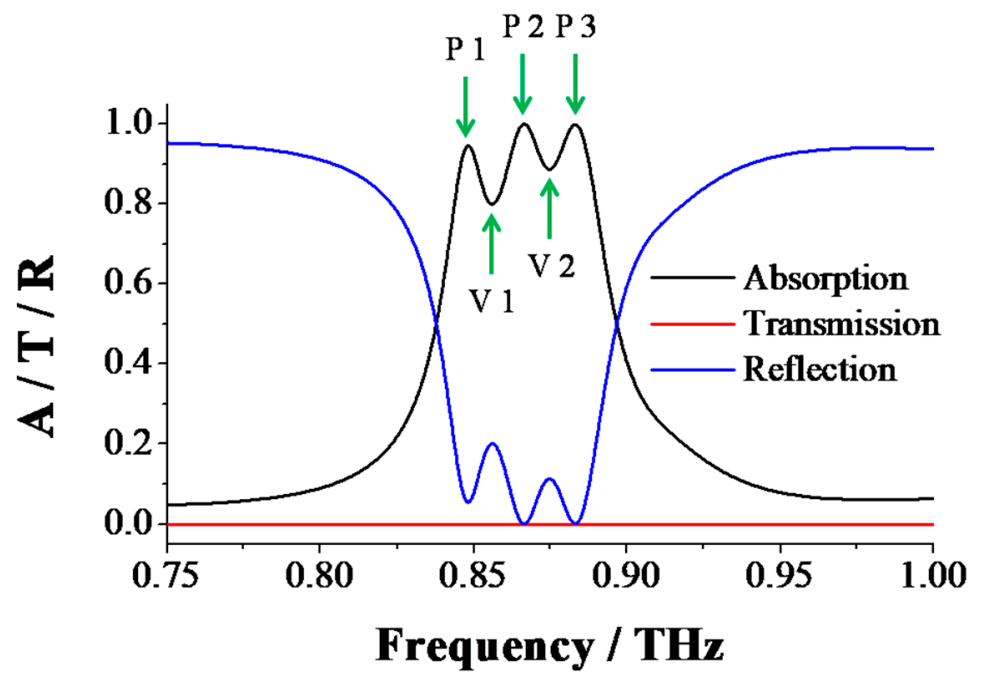

Figure 2. Simulated absorption/transmission/reflection spectra of our proposed wideband Mie resonance-based metasurface absorber. The absorption peaks 1, 2, and 3 at frequencies of $0.85 \mathrm{THz}$, $0.867 \mathrm{THz}$, and $0.883 \mathrm{THz}$ are marked with green arrows and labels "P1", "P2", and "P3", respectively. The absorption valley 1 and valley 2 at frequencies of $0.857 \mathrm{THz}$ and $0.875 \mathrm{THz}$ are marked with green arrows and labels "V1" and "V2", respectively. 


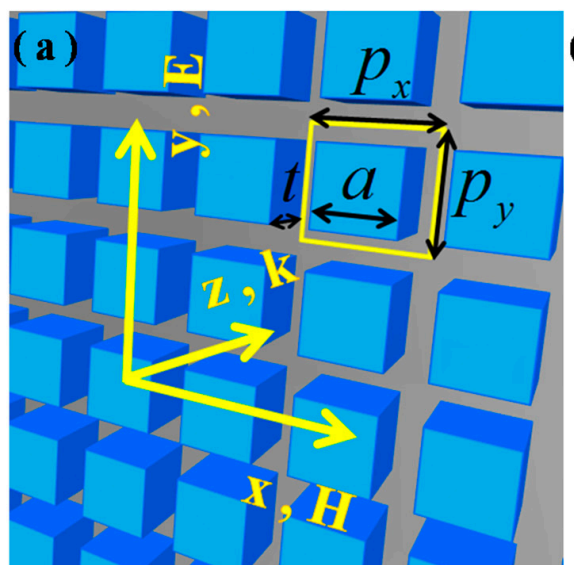

(c)

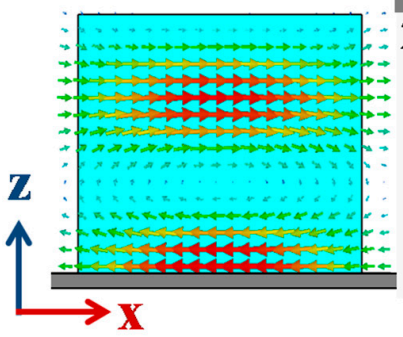

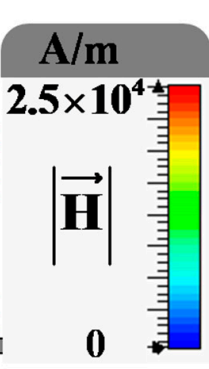

(b)

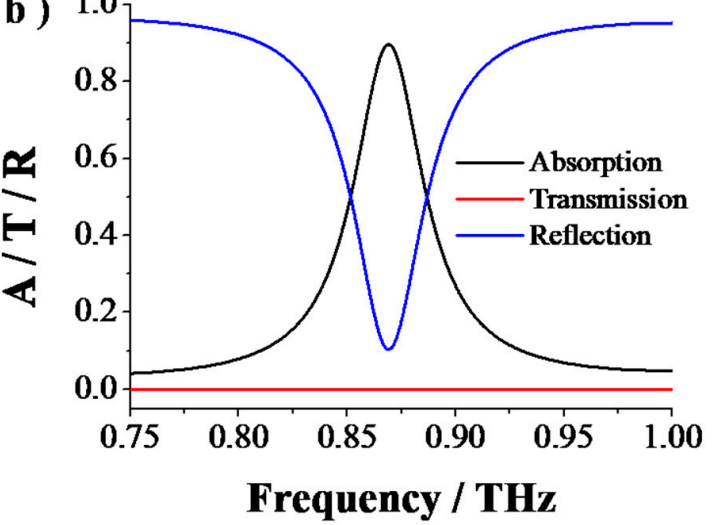

(d)

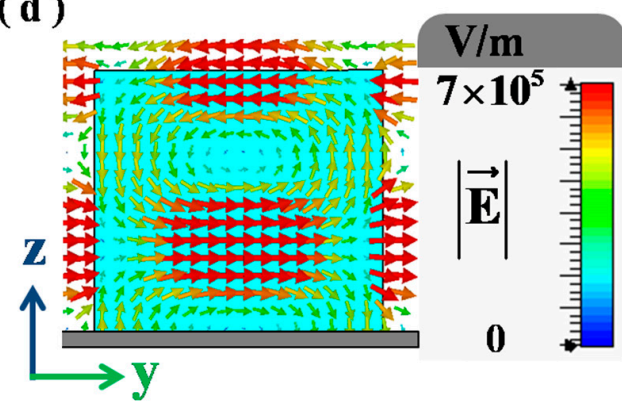

Figure 3. (a) Schematic diagram. (b) Simulated absorption/transmission/reflection spectra of the traditional metasurface with a single dielectric resonator in each unit cell. (c) The cross-sectional electric field distribution in the $y-z$ plane and (d) the cross-sectional magnetic field distribution in the $x-z$ plane for the unit cell of the traditional metasurface with a single dielectric resonator in each unit cell.

In order to better analyze this phenomenon, we then simulated the traditional Mie resonance-based metasurface. The schematic diagram is shown in Figure 3a. The dielectric blocks were arranged evenly and periodically on the aluminum substrate, with constant periods of both $p_{x}=p_{y}=40 \mu \mathrm{m}$. Each unit cell contained a single dielectric square block, whose sizes were $\mathrm{a}=33 \mu \mathrm{m}$, $t=30 \mu \mathrm{m}$. The simulation setup was the same as previously mentioned. Figure $3 \mathrm{~b}$ exhibits the absorption (black curve), transmission (red curve), and reflection (blue curve) spectra of the Mie resonance-based metasurface absorber with a single dielectric resonator in the unit cell. We can see that there was a reflection dip lower than 0.1 and an absorption peak of about 0.9 at around $0.869 \mathrm{THz}$, and the transmission was zero. We also simulated the electric field (E-field) and magnetic field (H-field) distributions of the single dielectric resonator at the frequency of the absorption peak. Cross-sectional H-field distribution in the $x-z$ plane is shown in Figure $3 c$, and the cross-sectional E-field distribution in the $y-z$ plane is shown in Figure $3 \mathrm{~d}$. At the frequency of $0.869 \mathrm{THz}$, the H-field was almost linearly parallel to the $x$-axis, and the vortical E-field was obviously distributed inside the dielectric block. This showed that a magnetic resonance occurred in the dielectric resonator at this frequency, illustrating that the absorption of this metasurface was related to the magnetic resonance.

As for our combined metasurface absorber, the electromagnetic field distribution at the frequencies of three absorption peaks (the frequencies of peaks 1, 2, 3 were $0.85 \mathrm{THz}, 0.867 \mathrm{THz}$, and $0.883 \mathrm{THz}$, respectively); these are shown in Figure $4 \mathrm{a}-\mathrm{c}$. Each part of Figure 4 displays the cross-sectional magnetic field distribution in the $x$-z plane with $y=40 \mu \mathrm{m}, 0 \mu \mathrm{m},-40 \mu \mathrm{m}$ and cross-sectional electric field distribution in the $y$-z plane with $x=-40 \mu \mathrm{m}, 0 \mu \mathrm{m}, 40 \mu \mathrm{m}$ for our combined metasurface absorber at different frequencies. At the absorption peaks 1, 2, 3, there were magnetic resonances, which were the same as the phenomenon inside the single block existing inside the third, second, and first row of squares, respectively. The magnetic resonance occurring in a smaller block corresponded to an absorption peak with a higher frequency. We also simulated the electromagnetic field distribution 
at the absorption dips between peaks 1 and 2 and peaks 2 and 3, as shown in Figure 5. We could observe that although there were linear magnetic field and vortical electric field inside the blocks, the phenomenon was different. In Figure $5 \mathrm{a}$, it can be seen that the strongest $\mathrm{H}$-field was located at the second row of blocks, while the third row of blocks had the strongest E-field. In Figure $5 b$, it can be seen that the strongest $\mathrm{H}$-field in the first row corresponded to the strongest electric field in the second row. This phenomenon indicates that there was a strong coupling between blocks at these two frequencies. All in all, the magnetic resonances occurring inside blocks of different sizes caused absorption peaks at different frequencies, while the special resonance caused by the near-field coupling between blocks led to relatively weaker absorption valleys between absorption peaks. Therefore, incorporating similar structures of different sizes into each unit cell could broaden the absorption bandwidth.
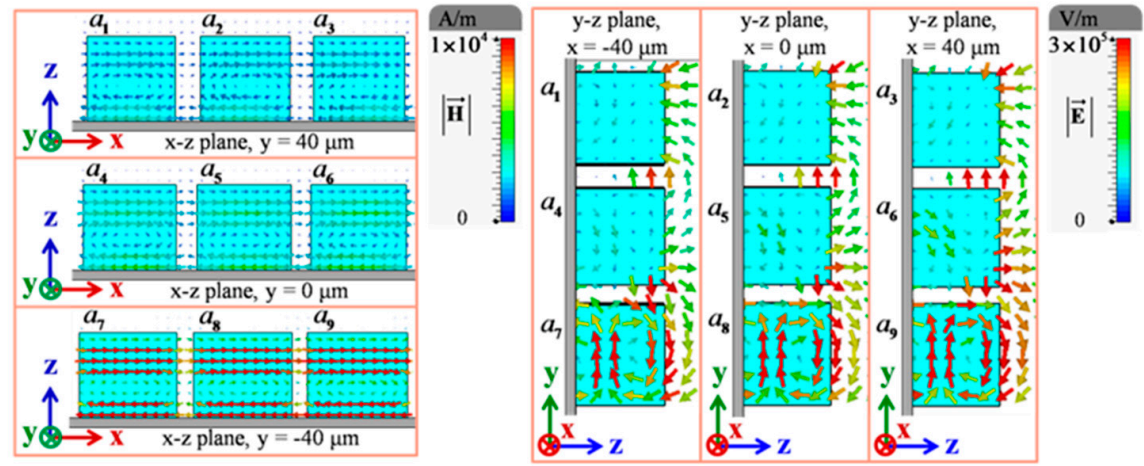

(a) Peak $1(0.85 \mathrm{THz})$
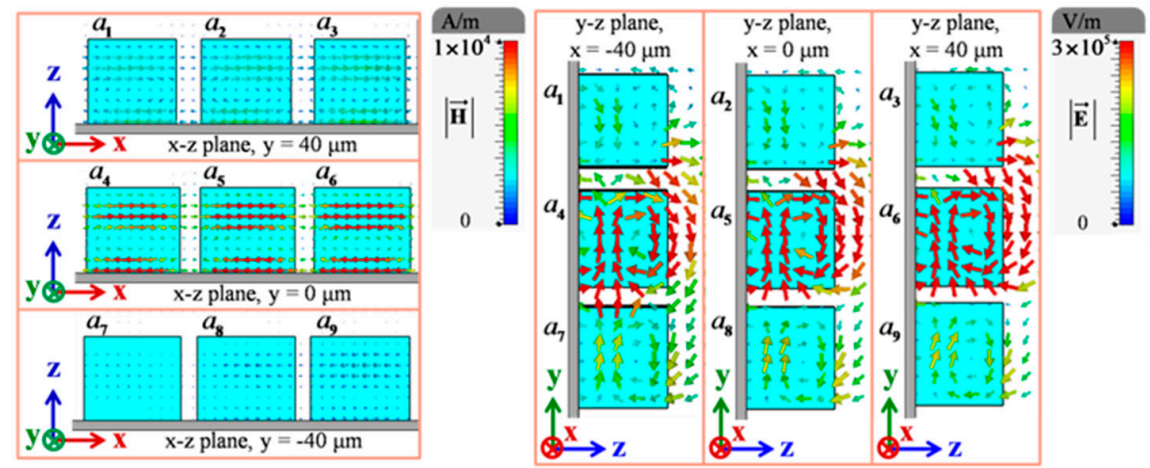

(b) Peak $2(0.867 \mathrm{THz})$
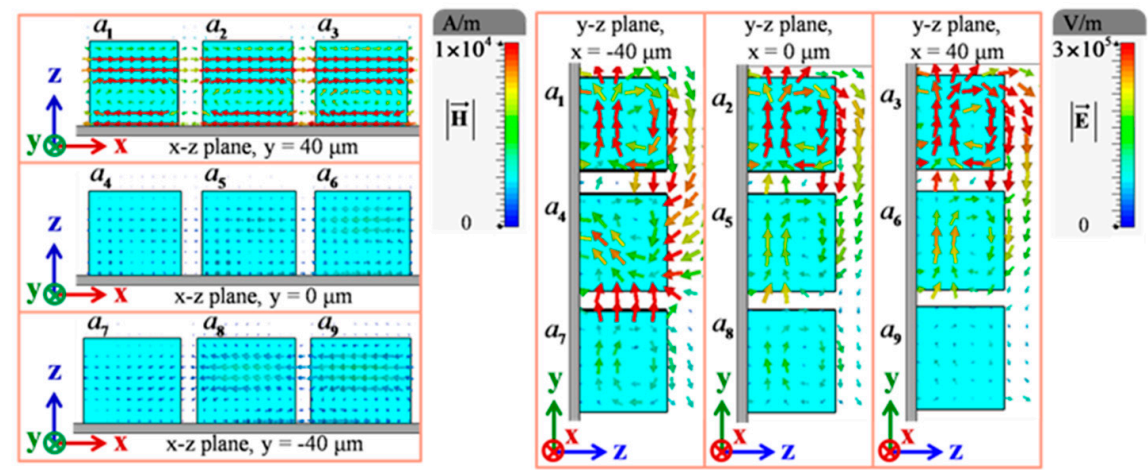

(c) Peak $3(0.883 \mathrm{THz})$

Figure 4. The cross-sectional magnetic field distribution in the $x-z$ plane with $y=40 \mu \mathrm{m}, 0 \mu \mathrm{m}$, $-40 \mu \mathrm{m}$ and cross-sectional electric field distribution in the $y-z$ plane with $x=-40 \mu \mathrm{m}, 0 \mu \mathrm{m}$, $40 \mu \mathrm{m}$ for our combined metasurface absorber at the frequency of (a) $0.85 \mathrm{THz}$; (b) $0.867 \mathrm{THz}$, and (c) $0.883 \mathrm{THz}$, respectively. 

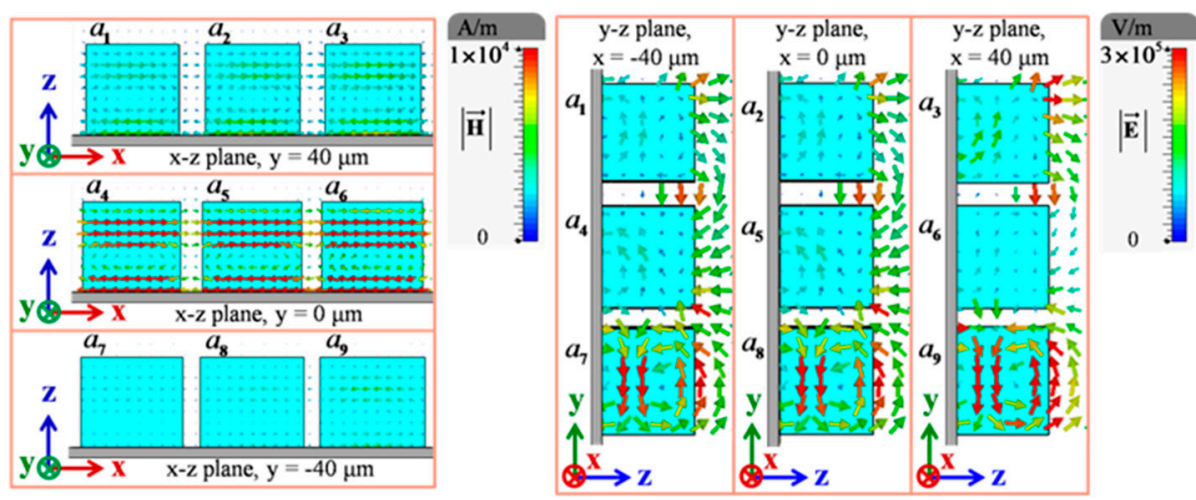

(a) Valley $1(0.857 \mathrm{THz})$
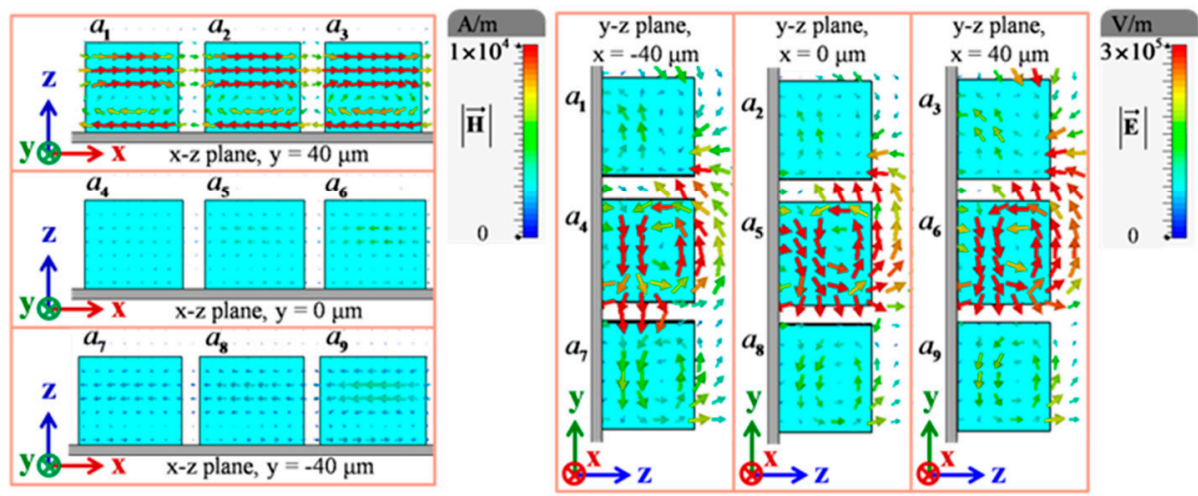

(b) Valley $2(0.875 \mathrm{THz})$

Figure 5. The cross-sectional magnetic field distribution in the $x$-z plane with $y=40 \mu \mathrm{m}, 0 \mu \mathrm{m},-40 \mu \mathrm{m}$ and the cross-sectional electric field distribution in the $y$ - $z$ plane with $x=-40 \mu \mathrm{m}, 0 \mu \mathrm{m}, 40 \mu \mathrm{m}$ for our combined metasurface absorber at the frequency of (a) $0.857 \mathrm{THz}$ and (b) $0.875 \mathrm{THz}$.

We compared the absorption spectra of the metasurface absorber with single dielectric resonator-four or nine dielectric resonators in one unit cell-as shown in Figure 6a. Bandwidth when absorption rate over 0.8 is also marked out. The size of this single dielectric resonator was $33 \times 33 \times 30 \mathrm{\mu m}^{3}$. These four resonators of the combined metasurface absorber were $32.1 \times 32.1 \times 30 \mu \mathrm{m}^{3}, 32.7 \times 32.7 \times 30 \mu \mathrm{m}^{3}, 33.3 \times 33.3 \times 30 \mu \mathrm{m}^{3}$, and $33.9 \times 33.9 \times 30 \mu \mathrm{m}^{3}$. The combined metasurface absorber with nine resonators was the same as mentioned above. As we can see, as the number of different resonators increased in each unit cell, the frequency domain of absorption also widened significantly. The bandwidth of absorption rate above 0.8 was $0.012 \mathrm{THz}$, $0.024 \mathrm{THz}$, and $0.047 \mathrm{THz}$. Figure $6 \mathrm{~b}$ shows the absorption spectra of the single resonator metasurface with different side lengths (from $31 \mu \mathrm{m}$ to $35 \mu \mathrm{m}$ ). Comparing Figures $6 \mathrm{a}$ and $6 \mathrm{~b}$, we can see that the absorption bandwidth of the metasurface combining nine resonators was comparable with the bandwidth covered by nine adjacent absorption spectra (Figure 6b), which was the same as the four blocks combined. Moreover, according to Figure 6, we can also observe that the greater the number of combined structures (or the larger the range of the structure size), the wider was the absorption frequency range of this combined metasurface absorber. 


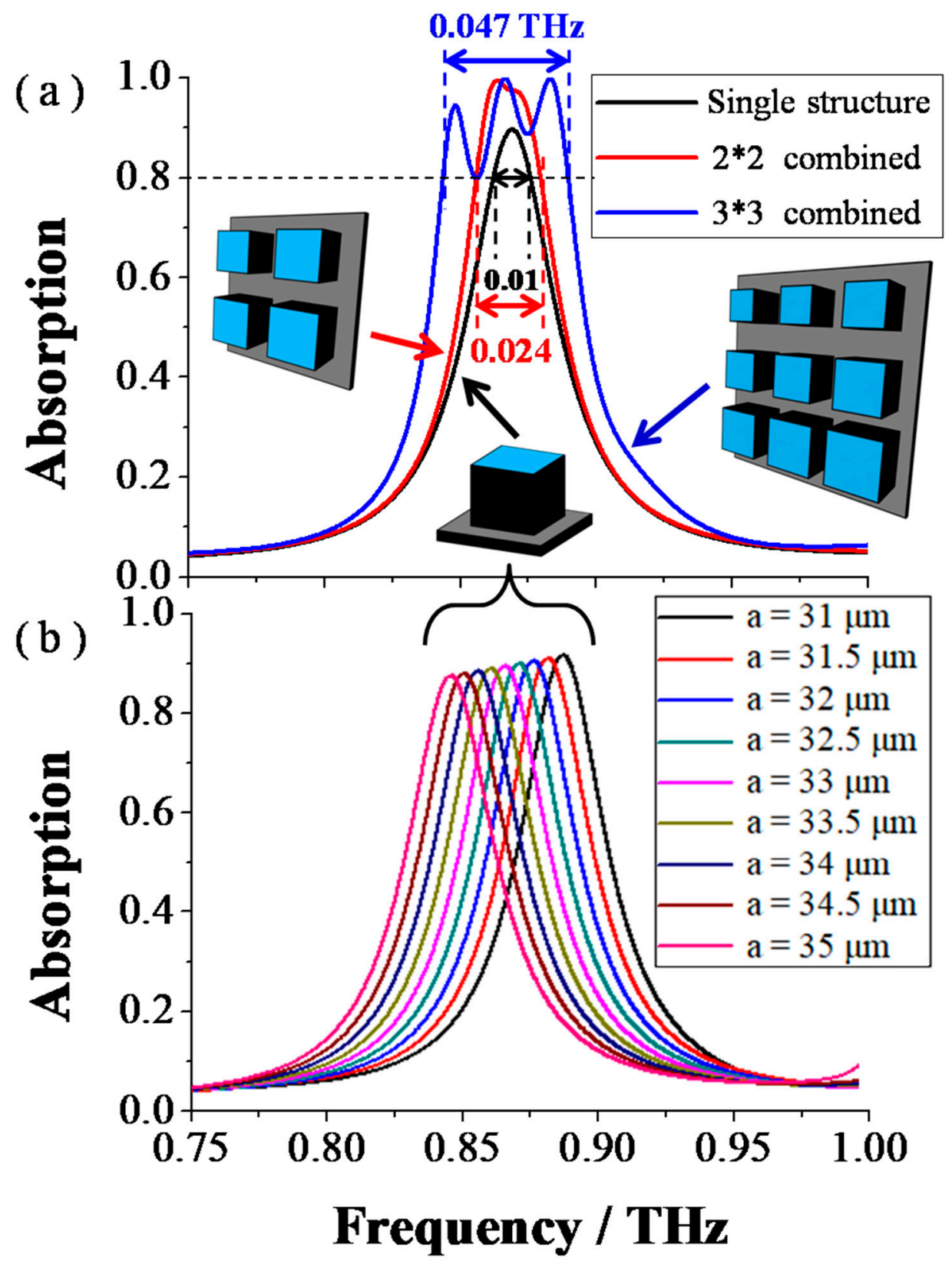

Figure 6. (a) Absorption spectra of the Mie resonance-based metasurface absorber with one, four, and nine dielectric resonator in one unit cell. (b) Absorption spectra of the traditional metasurface absorber with single dielectric resonator (side lengths from $31 \mu \mathrm{m}$ to $35 \mu \mathrm{m}$ ) in one unit cell.

It is well known that the dielectric resonator can restrict the electromagnetic field into its structure, which is also the basis of silicon-based waveguides. In this design, when the size of the block (the proportion of the block in the unit cell) is slightly reduced, the electromagnetic energy will be limited to a smaller area, and the resonance inside the structure will be stronger due to higher energy density. We checked the electromagnetic field of different sizes and found that the maximum magnetic field amplitude of the smaller structure would be larger. For example, the maximums of the magnetic field distribution were $40,491 \mathrm{~A} / \mathrm{m}, 38,070 \mathrm{~A} / \mathrm{m}$, and 36,051 A/m when the side lengths of the single block structure were $31 \mu \mathrm{m}, 33 \mu \mathrm{m}$, and $35 \mu \mathrm{m}$, respectively. This absorber was based on the magnetic resonance of the dielectric structure and was mainly affected by the magnetic field energy. Thus, the smaller size corresponded to higher absorption rate, and there was a slope in the absorption level for different sizes. It is worth noting that this phenomenon is based on the premise that structural changes do not break the resonance. 


\section{Further Investigation on Different Incident Angles and Polarization States}

Next, we explored the influence of the polarization state and the incident angle of incident light on the performance of our combined metasurface absorber. Figure $7 \mathrm{a}$ is the schematic diagram of the irradiation of incident polarized light. The three red arrows represent the $x$-axis, the $y$-axis, and the $z$-axis, while the two green arrows represent the polarization direction of the magnetic field and the direction of the incident light. $\varphi$ is the angle between the polarization direction of the magnetic field and the $x$-axis, while $\theta$ is the angle between the direction of the incident light and the opposite direction of the $z$-axis in the $x-z$ plane. Figure $7 \mathrm{~b}$ displays the absorption spectra at different polarization of normally incident light. When $\varphi=0^{\circ}$, our combined metasurface absorber had the widest absorption bandwidth. However, as $\varphi$ became larger, the absorption bandwidth of the wave absorber became narrower. The bandwidths at $80 \%$ absorption for the combined metasurface were $0.047,0.045,0.038$, 0.022 , and $0.018 \mathrm{THz}$ when $\varphi$ equaled $0^{\circ}, 22.5^{\circ}, 45^{\circ}, 67.5^{\circ}, 90^{\circ}$, respectively. The appearance of this phenomenon may be related to the fact that this metasurface absorber is based on magnetic resonance. When the magnetic field was along the $y$ direction (the direction in which the side lengths of the resonators increase jumpingly, for example, $a_{1}-a_{4}-a_{7}$ ), the absorption bandwidth was reduced to $40 \%$. Despite this, the narrowest absorption bandwidth of our combined metasurface absorber was still nearly twice that of a single resonator. This proves that our combined metasurface absorber can effectively broaden the absorption bandwidth regardless of the polarization of the incident light. Figure 7c shows the absorption spectra at different incident angle of the incident light when $\varphi=0^{\circ}$. When the light was normally incident, the absorption effect was the best. As the incident light gradually became tilted, the absorption rate slightly decreased, while the absorption bandwidth was almost constant. Even when the light was incident at a $45^{\circ}$ angle, the absorption rate could still reach more than $75 \%$. This shows that our combined metasurface absorber can still work well within a certain range of oblique incident angles.

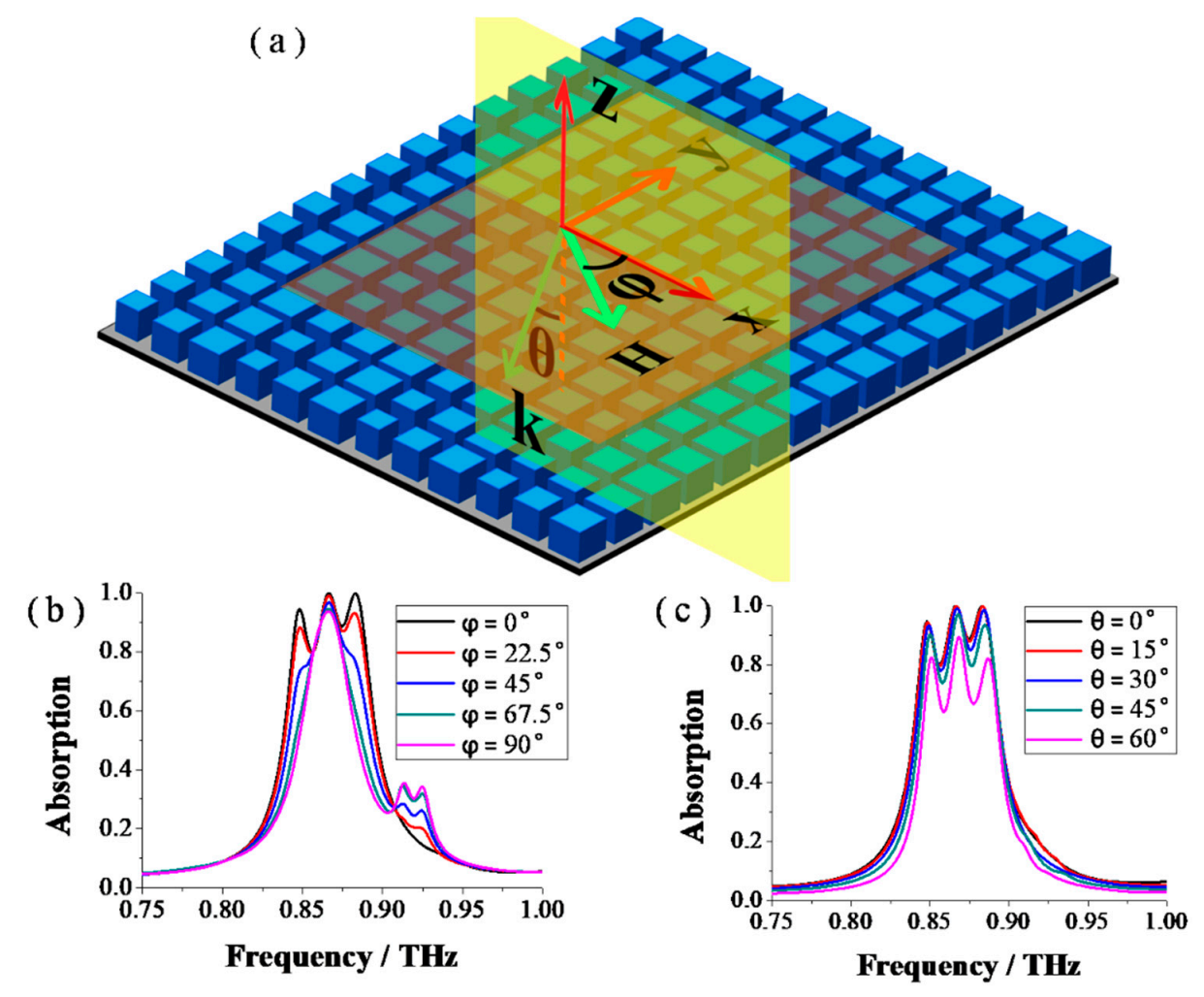

Figure 7. (a) Schematic diagram and absorption spectra of our wideband metasurface absorber consisting of nine dielectric resonators with different side lengths in one unit cell, (b) at different polarization angle, and (c) at different incident angle of the incident light. 
We can see that in Figure 7b, there are two little absorption peaks around $0.91 \mathrm{THz}$ and $0.923 \mathrm{THz}$. We simulated the electromagnetic field distributions at these two frequencies, as shown in Figures 8 and 9. In Figure 8, it can be seen that vortical E-fields were mainly distributed inside the dielectric blocks of $\mathrm{a}_{2}-\mathrm{a}_{6}$, while the H-fields inside the blocks of $\mathrm{a}_{1}, \mathrm{a}_{7} \sim \mathrm{a}_{9}$ were almost linearly parallel to the $y$ axis. In Figure 9, vortical E-fields were mainly distributed inside the dielectric blocks of $a_{1}-a_{3}$, while the $\mathrm{H}$-fields inside the blocks of $\mathrm{a}_{4}-\mathrm{a}_{9}$ were almost linearly parallel to the $y$-axis. At these two frequencies, there was a common feature that the linearly magnetic fields in some blocks excited the vortical electric field in other blocks. If you look at these nine pieces as a whole, this phenomenon is somewhat similar to higher-order mode. This special mode-due to near-field coupling-leads to additional small absorption peaks.

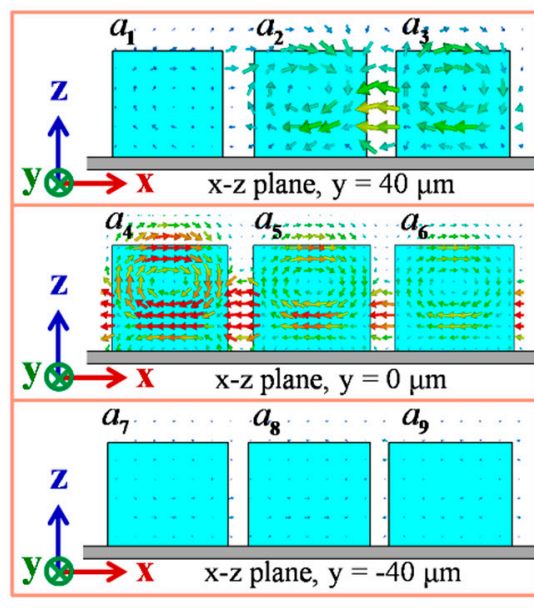

(a)
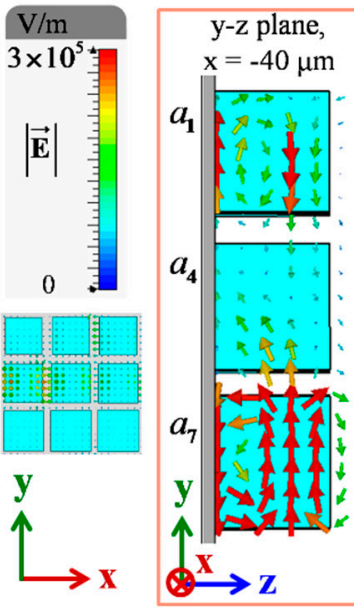
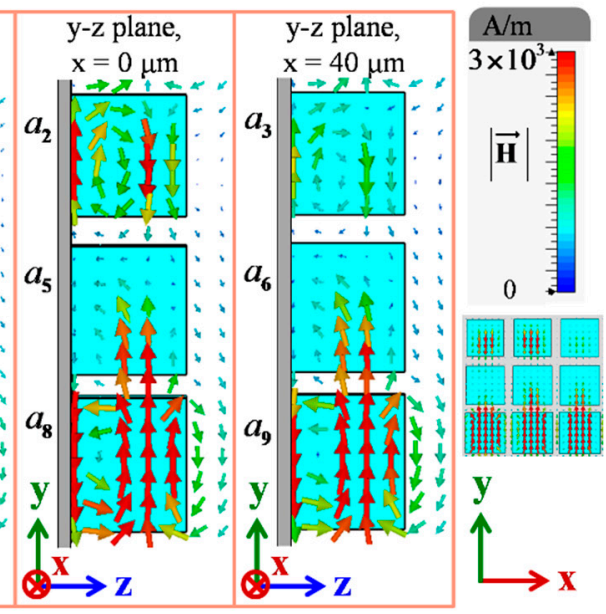

(b)

Figure 8. (a) The cross-sectional electric field distributions in the $x$ - $z$ plane with $y=40 \mu \mathrm{m}, 0 \mu \mathrm{m}$, $-40 \mu \mathrm{m}$ and (b) the cross-sectional magnetic field distribution in the $y$ - $z$ plane with $x=-40 \mu \mathrm{m}, 0 \mu \mathrm{m}$, $40 \mu \mathrm{m}$ for our combined metasurface absorber at the frequency of $0.91 \mathrm{THz}$. The electromagnetic field distributions in the $x-y$ plane at the height of $z=20 \mu \mathrm{m}$ are also shown below the color bar.

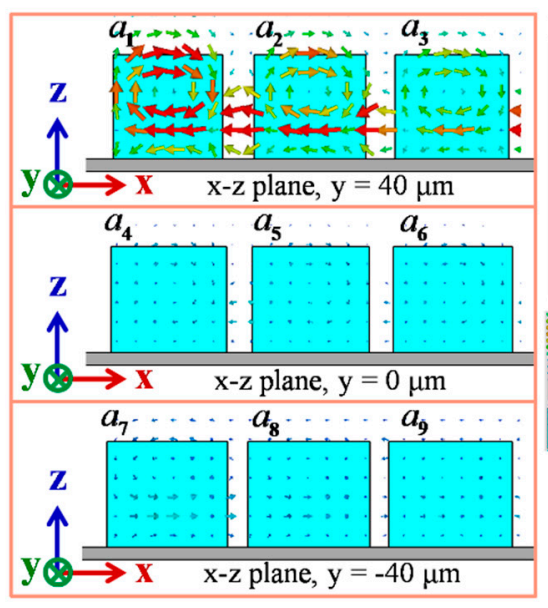

(a)
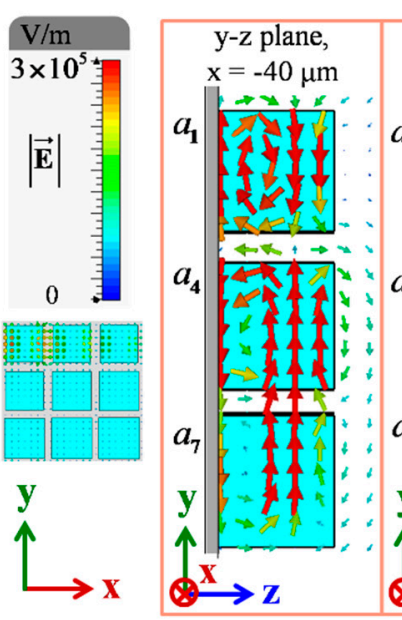

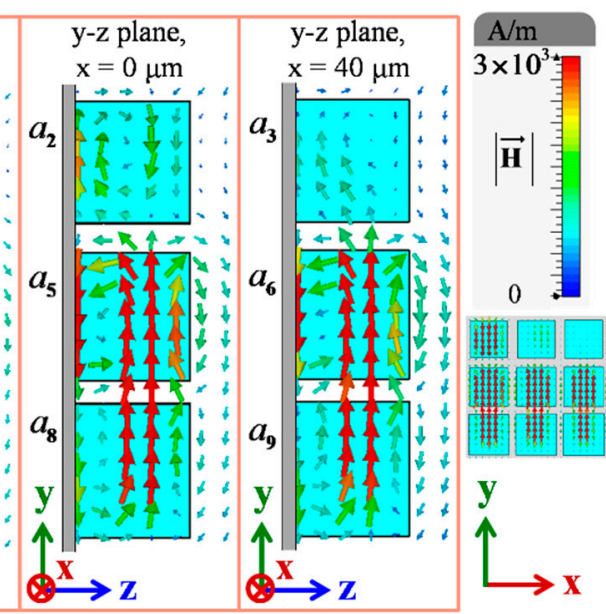

(b)

Figure 9. (a) The cross-sectional electric field distribution in the $x-z$ plane with $y=40 \mu \mathrm{m}, 0 \mu \mathrm{m},-40 \mu \mathrm{m}$ and (b) the cross-sectional magnetic field distribution in the $y$ - $z$ plane with $x=-40 \mu \mathrm{m}, 0 \mu \mathrm{m}, 40 \mu \mathrm{m}$ for our combined metasurface absorber at frequency of $0.923 \mathrm{THz}$. The electromagnetic field distributions in the $x-y$ plane at the height of $z=20 \mu \mathrm{m}$ are also shown below the color bar. 
The fabrication of our combined metasurface absorber is underway. Firstly, a $500 \mathrm{~nm}$ thick aluminum with a $10 \mathrm{~nm}$ thick chromium adhesion layer will be deposited on a quartz substrate by the electron-beam evaporator. Subsequently, samples will be coated again with a $10 \mathrm{~nm}$ of chromium via electron beam evaporation to avoid charging effects during the writing process. Then, referring to the method in Reference [30], a layer of titanium dioxide will be deposited on the sample by the atomic layer deposition (ALD) method. The deposition of the $\mathrm{TiO}_{2}$ can also be realized by utilizing chemical vapor deposition (CVD) [31]. The deposition rate is about $100 \mathrm{~nm} / \mathrm{s}$. Finally, a sequential process of lithography, mask deposition, lift-off, and reactive ion etching (RIE) can be utilized to obtain the desired structure. On the other hand, the material of the structure is not limited to titanium dioxide, other dielectric with high refractive index is also feasible to deposit on the metal film.

\section{Conclusions}

In summary, we propose a combined metasurface consisting of an aluminum substrate and an array of $\mathrm{TiO}_{2}$ blocks to achieve a wideband terahertz absorber. During our experiments, we found that the absorption bandwidth with this design was nearly 2.5 times and 5 times wider than a traditional single block when combining four and nine blocks, respectively. The widening multiple could be further improved by increasing the number of combinations in structures (variable parameters included number, spacing, dimensions etc.). We also investigated the effect of the incident angle and polarization on the performance of the absorber. The absorption bandwidth could be widened effectively in any polarization; when the incident angle was $45^{\circ}$, the absorption rate could still reach $75 \%$ or more.

Author Contributions: J.H. designed the structure and performed the numerical simulations; T.L. put forward the idea, analyzed the data, and wrote the article; C.S. and L.S. checked the spelling, grammar of this article and put forward some comments.

Funding: This research was funded by Public Projects of Zhejiang Province (LGG18F050003), National major scientific research instrument development project of Natural Science Foundation of China (61727816), and the National Natural Science Foundation of China (No. 61475128 and No. 61875251).

Acknowledgments: This work was supported by Public Projects of Zhejiang Province (LGG18F050003), National major scientific research instrument development project of Natural Science Foundation of China (61727816), and the National Natural Science Foundation of China (No. 61475128 and No. 61875251).

Conflicts of Interest: The authors declare no conflict of interest.

\section{References}

1. Dincer, F.; Akgol, O.; Karaaslan, M.; Ünal, E.; Sabah, C. Polarization Angle Independent Perfect Metamaterial Absorbers for Solar Cell Applications in the Microwave, Infrared, and Visible Regime. Prog. Electromagn. Res. 2014, 144, 93-101. [CrossRef]

2. Rufangura, P.; Sabah, C. Design and characterization of a dual-band perfect metamaterial absorber for solar cell applications. J. Alloy. Compd. 2016, 671, 43-50. [CrossRef]

3. Akhlaghi, M.K.; Schelew, E.; Young, J.F. Waveguide integrated superconducting single-photon detectors implemented as near-perfect absorbers of coherent radiation. Nat. Commun. 2015, 6, 8233. [CrossRef] [PubMed]

4. Mei, P.; Lin, X.Q.; Yu, J.W.; Boukarkar, A.; Zhang, P.C.; Yang, Z.Q. Development of a Low Radar Cross Section Antenna with Band-Notched Absorber. IEEE Trans. Antennas Propag. 2018, 66, 582-589. [CrossRef]

5. Sui, S.; Ma, H.; Wang, J.; Pang, Y.; Feng, M.; Xu, Z.; Qu, S. Absorptive Coding Metasurface for Further Radar Cross Section Reduction. J. Phys. D Appl. Phys. 2017, 51, 065603. [CrossRef]

6. Liu, X.; Gao, J.; Yang, H.; Wang, X.; Guo, C. Multiple infrared bands absorber based on multilayer gratings. Opt. Commun. 2018, 410, 438-442. [CrossRef]

7. Bian, L.A.; Yang, L.; Liu, P.; Chen, Y.; Liu, H.; Zhou, Q. Controllable perfect absorption in a double-cavity photonic crystal with one graphene monolayer. J. Phys. D Appl. Phys. 2017, 51, 025106. [CrossRef]

8. Holloway, C.L.; Kuester, E.F.; Gordon, J.A.; O'Hara, J.; Booth, J.; Smith, D.R. An Overview of the Theory and Applications of Metasurfaces: The Two-Dimensional Equivalents of Metamaterials. IEEE Antennas Propag. Mag. 2012, 54, 10-35. [CrossRef] 
9. Kildishev, A.V.; Shalaev, V.M. Planar Photonics with Metasurfaces. Science 2013, 339, 1232009. [CrossRef] [PubMed]

10. Yu, N.; Capasso, F. Flat optics with designer metasurfaces. Nat. Mater. 2014, 13, 139-150. [CrossRef] [PubMed]

11. Yu, N.; Genevet, P.; Kats, M.A.; Aieta, F.; Tetienne, J.P.; Capasso, F.; Gaburro, Z. Light propagation with phase discontinuities: Generalized laws of reflection and refraction. Science 2011, 334, 333-337. [CrossRef] [PubMed]

12. Dong, G.; Shi, H.; Xia, S.; Zhang, A.; Xu, Z.; Wei, X. Ultra-broadband perfect cross polarization conversion metasurface. Opt. Commun. 2016, 365, 108-112. [CrossRef]

13. Spinelli, P.; Verschuuren, M.A.; Polman, A. Broadband omnidirectional antireflection coating based on subwavelength surface Mie resonators. Nat. Commun. 2012, 3, 692. [CrossRef] [PubMed]

14. Pineda, J.D.D.; Mitchell-Thomas, R.C.; Hibbins, A.P.; Sambles, J.R. A broadband metasurface Luneburg lens for microwave surface waves. Appl. Phys. Lett. 2017, 111, 211603. [CrossRef]

15. Gong, J.; Yang, F.; Zhang, X. A novel wideband optical absorber based on all-metal 2D gradient nanostructures. J. Phys. D Appl. Phys. 2017, 50, 455105. [CrossRef]

16. Li, H.; Qi, L.; Zhang, X.; Wei, Z. Metamaterial for polarization-incident angle independent broadband perfect absorption in the terahertz range. Opt. Mater. Express 2017, 7, 3294-3302. [CrossRef]

17. Luo, M.; Zhou, Y.; Wu, S.; Chen, L.S. Wide-angle broadband absorber based on one-dimensional metasurface in the visible region. Appl. Phys. Express 2017, 10, 092601. [CrossRef]

18. Ghasemi, M.; Choudhury, P.K.; Baqir, M.A.; Zain, A.R.M.; Majlis, B.Y. Metamaterial absorber comprising chromium-gold nanorods-based columnar thin films. J. Nanophotonics 2017, 11, 043505. [CrossRef]

19. Mou, J.; Shen, Z. Broadband and thin magnetic absorber with non-Foster metasurface for admittance matching. Sci. Rep. 2017, 7, 6922. [CrossRef] [PubMed]

20. Bhattarai, K.; Silva, S.; Song, K.; Urbas, A.; Lee, S.J.; Ku, Z.; Zhou, J. Metamaterial Perfect Absorber Analyzed by a Meta-cavity Model Consisting of Multilayer Metasurfaces. Sci. Rep. 2017, 7, 10569. [CrossRef] [PubMed]

21. Sun, L.; Padilla, W.J.; Ming, X.; Liu, X. Degenerate critical coupling in all-dielectric metasurface absorbers. Opt. Express 2017, 25, 24658-24669. [CrossRef]

22. Zhou, Z.; Chen, K.; Zhao, J.; Chen, P.; Jiang, T.; Zhu, B.; Feng, Y.; Li, Y. Metasurface Salisbury screen: Achieving ultra-wideband microwave absorption. Opt. Express 2017, 25, 30241-30252. [CrossRef] [PubMed]

23. Sun, Z.; Zhao, J.; Zhu, B.; Jiang, T.; Feng, Y. Selective wave-transmitting electromagnetic absorber through composite metasurface. AIP Adv. 2017, 7, 115017. [CrossRef]

24. Kenney, M.; Grant, J.; Shah, Y.D.; Escorcia-Carranza, I.; Humphreys, M.; Cumming, D.R.S. Octave-Spanning Broadband Absorption of Terahertz Light using Metasurface Fractal-Cross Absorbers. ACS Photonics 2017, 4, 2604-2612. [CrossRef]

25. Liao, Z.; Gong, R.; Nie, Y.; Wang, T.; Wang, X. Absorption enhancement of fractal frequency selective surface absorbers by using microwave absorbing material based substrates. Photonics Nanostruct. 2011, 9, 287-294. [CrossRef]

26. Faruque, M.R.I.; Hasan, M.M.; Islam, M.T. Tree-shaped fractal meta-surface with left-handed characteristics for absorption application. Appl. Phys. A Mater. 2018, 124, 127. [CrossRef]

27. Zhu, L.; Dong, L. Electromagnetically induced transparency with wide band in all-dielectric microstructure based on Mie resonances. J. Opt. 2014, 16, 125105. [CrossRef]

28. Sui, C.; Li, X.; Lang, T.; Jing, X.; Liu, J.; Hong, Z. High Q-Factor Resonance in a Symmetric Array of All-Dielectric Bars. Appl. Sci. 2018, 8, 161. [CrossRef]

29. Wang, Y.; Li, X.; Lang, T.; Jing, X.; Hong, Z. Multiband guided-mode resonance filter in bilayer asymmetric metallic gratings. Opt. Laser Technol. 2018, 103, 135-141. [CrossRef] 
30. Devlin, R.C.; Khorasaninejad, M.; Chen, W.T.; Oh, J.; Capasso, F. Broadband high-efficiency dielectric metasurfaces for the visible spectrum. Proc. Natl. Acad. Sci. USA 2016, 113, 10473-10478. [CrossRef] [PubMed]

31. Sun, H.; Wang, C.; Pang, S.; Li, X.; Tao, Y.; Tang, H.; Liu, M. Photocatalytic $\mathrm{TiO}_{2}$ films prepared by chemical vapor deposition at atmosphere pressure. J. Non-Cryst. Solids 2008, 354, 1440-1443. [CrossRef] 